\title{
Analytical Simulation of Two Dimensional Advection Dispersion Equation of Contaminant Transport
}

\section{${ }^{* 1}$ COLE, AT $;{ }^{2}$ ABDULRAHIM, A $;{ }^{3}$ OLAYIWOLA, RO; ${ }^{4}$ SHEHU, MD}

\author{
1,2,3,4 Department of Mathematics, \\ Federal University of Technology, Minna, \\ E-mail: olayiwolarasaq13@gmail.com
}

\begin{abstract}
The study was designed to investigate the analytical simulation of two dimensional advection dispersion equation of contaminant transport. The steady state flow condition of the contaminant transport where inorganic contaminants in aqueous waste solutions are disposed of at the land surface where it would migrate through the verdoze zone to underground water is considered. We solved the two dimensional advection dispersion equation analytically which is solute transport model without sorption or degradation using change of variable method. We critically reviewed two dimensional equations depicting the transport of contaminant in groundwater and investigate with the help of graphical representation the effect of Peclet number on the concentration of contaminant and established real life interpretation of contaminant transport. Two cases were considered, when Peclet number is less than one and when Peclet number is greater than one. The result obtained revealed that the contaminant concentration increases along $\mathrm{x}$ direction and decreases along y direction for both values of peclet number greater than one and less than one. The study has contributed to knowledge through the method utilized to achieve the model analytical solution and the Physical interpretation of Peclet number. From the analysis, we recommend for further studies on the contaminant transport which also depends on the available data, that the extension of advection -dispersion model to three dimensions and comparison of travel time of contaminant transport solution to Kinetic or multi-component mode.C JASEM

https://dx.doi.org/10.4314/jasem.v21i5.6
\end{abstract}

Keywords Contaminant, Seepage Velocity, Aquifer, Advection-dispersion Equation, change of variable methods

The most usual way of "managing" town refuses is achieve by depositing it in sanitary landfills. In moist and semi-humid region, the movement of contaminants from the refuse into the ground water zone lying underneath normally occur due to infiltration through landfills, the zones of contaminated ground water expand many hundreds meters. In a few circumstances, contaminant can lead to critical deterioration of aquifer used for ground water source; therefore, we need to know how contaminants moves from place at which they are created, leading to impacts, which can be far from the contaminant source. On the other hand, some contaminant, such as sewage sludge, can be degrading in the environment if they are sufficiently dilute. For these contaminants, slow transport slow dilution can result in excessively high contaminant concentrations, with resulting increased adverse impacts. It is therefore important to have a simple method of making contaminant transport calculations for contaminant at concentration other than the average (for contaminant fronts) or the peak (for contaminant pulse). The steady state flow condition of the contaminant transport in a point source where inorganic contaminants in aqueous waste solutions are disposed of at the land surface must migrate through the verdoze zone to underground water is considered.
It known here that, if a model is developed and used in application to a problem, the solution will be analyses and interpreted with regard to the problem in question. Aiyesimi, (2004) studied the mathematical analysis of environmental contamination of the Freudlich non- linear contaminant transport formulation. Gideon and Aiyesimi (2005) lucubrated on the non-linear contaminant flow and the influence of retardation factor on it.

Mulligan et al, (1999) Groundwater flow below surface layer is pushed by difference in energy water flows from elevated-energy regions to ground energy regions. The energy component of a unit volume of water is ascertained by the addition of gravitational potential energy, kinetic energy, and pressure energy.

Olayiwola et al, (2014) presents 2D mathematical model describing the transport of a conservation contaminant through a homogeneous finite aquifer under transient flow. They assume the aquifer is subject to contamination because of the timeindependent source concentration. Both the sinusoidal changing and exponentially reducing forms of seepage velocity are observed for studying seasonal variation problems. Adeboye et al (2013), formulated analysis of contaminant take over in a system of aquifer, expressing the character of 
contaminants for various values of $\alpha, b$ and $\gamma$. They observed that for different values of $\alpha, b$ and $\gamma$ the level of contamination reduces over the domain of a uniform source of contamination at $c^{u}(x)=1$.

The aim of the study is to derive analytical solution of two dimensional equations and simulation of contaminant transport in underground water. Critically review two - dimensional equation depicting the transport of contaminant in groundwater. Solve the equation analytically using change of variable method and direct integration.

The relation of these processes with one another can be expressed mathematically as follows:

$$
\begin{aligned}
& R \frac{\partial c}{\partial t}=\frac{\partial}{\partial x_{i}}\left(D_{i j} \frac{\partial c}{\partial x_{i}}\right)-\frac{\partial}{\partial x_{i}}\left(c v_{i}\right)-\frac{c^{\prime} w^{\prime}}{n} \\
& v_{i}=\frac{-k_{i j}}{n} \frac{\partial h}{\partial_{x, j}} \\
& R=\left(1+\frac{\rho_{b} k_{d}}{n}\right),
\end{aligned}
$$

Where;

$\mathrm{c}=$ Contaminant Transport

$v_{i}=$ Seepage or Average pore water Velocity in the direction $x_{i}$

$D_{i j}=$ Dispersion Coefficient

$k_{i j}=$ Hydraulic Conductivity

$c^{\prime \prime}=$ Solute Concentration in the source or sink fluid

$w=$ Volume Flow rate per unit volume of the source or sink

$n=$ Effective Porosity

$h=$ Hydraulic Head

$\mathrm{R}=$ Retardation

$x_{i}=$ Cartesian Coordinate

An evaluated two-dimensional form shows the contaminant transport in groundwater in an isotropic, homogeneous medium having a steady-state flow that is unidirectional with seepage velocity $\mathrm{v}$ is given as:

$$
R \frac{\partial c}{\partial t}=D_{L} \frac{\partial^{2} c}{\partial x^{2}}+D_{T} \frac{\partial^{2} c}{\partial y^{2}}-v \frac{\partial c}{\partial x}
$$

Where; investigate with the help of graphical representation the effects of Peclet number on the concentration of contaminant. vi. Establish real life interpretation of the contaminant transport

Mathematical formulation: Patil and Chole (2014), forecast the environmental repercussion of contamination of groundwater, there should be information as to where there will be interference of the contaminant, the time it will arrive and the concentrations potential. The introduction of contaminant into groundwater is by: (i) advection due to groundwater flow; (ii) dispersion due to molecular diffusion and mechanical mixing; and (iii) retardation due to adsorption.

$D_{i j}=$ Dispersion Coefficient

$k_{i j}=$ Hydraulic Conductivity

$c=$ Contaminant Concentration

$v=$ Seepage or Average pore water velocity volume

$D_{L}=$ Coefficient of Longitudinal Dispersion

$D_{T}=$ transversal dispersion coefficient

$\mathrm{R}=$ retardation

Since sorption onto the sediment reduces the apparent advective - dispersive flux by a factor equal to $R$. The sorption considered negligible, in which case $k_{d}=0$, and the value for $R$ becomes equal to one and the equation (3.4) reduces to:

$$
\frac{\partial c}{\partial t}=D_{L} \frac{\partial^{2} c}{\partial x^{2}}+D_{T} \frac{\partial^{2} c}{\partial y^{2}}-v \frac{\partial c}{\partial x}
$$

(5)

The initial and boundary conditions are formulated as follows:

$$
\left.\begin{array}{l}
c(x, y, t)=0 \quad x \geq 0, y \geq 0, t=0 \\
c(x, y, t)=c_{0} \quad x=0, y=0, t>0 \\
c(x, y, t)=0 \quad \text { as } x^{2}+y^{2} \rightarrow \infty, t>0
\end{array}\right\}
$$

Steady

state, with

$D_{L}=D_{T}=D$ and $v=$ cons $\tan t$, we have:

$v \frac{\partial c}{\partial x}=D\left(\frac{\partial^{2} c}{\partial x^{2}}+\frac{\partial^{2} c}{\partial y^{2}}\right)$

Together with boundary conditions:

$$
\left.\begin{array}{l}
c(x, y)=c_{0} \quad \text { on } x=0, y=o \\
c(x, y) \rightarrow 0 \quad \text { as } x^{2}+y^{2} \rightarrow \infty
\end{array}\right\}
$$


We non-dimensionalised equations (7) and (8) using the following variables which are dimensionless:

$x^{\prime}=\frac{x}{L}, \quad y^{\prime}=\frac{y}{L}, \quad \phi=\frac{c}{c_{0}}$

dropping prime we obtain

$\frac{\partial \phi}{\partial x}=\frac{1}{p_{e m}}\left(\frac{\partial^{2} \phi}{\partial x^{2}}+\frac{\partial^{2} \phi}{\partial y^{2}}\right)$

Together with boundary conditions:

after

$$
\left.\begin{array}{l}
\phi(x, y)=1 \quad \text { on } x=0, y=0 \\
\phi(x, y) \rightarrow 0 \\
\text { as } x^{2}+y^{2} \rightarrow \infty
\end{array}\right\}
$$

Where,

$$
\mathrm{P}_{\mathrm{em}}=\frac{L V}{D}
$$

\section{Analytical Solution}

By change of variable method,

Let

$\phi(x, y)=\phi(\eta), \quad \eta=\left(\frac{\left(x^{2}+y^{2}\right)^{\frac{1}{2}}-x}{2 \epsilon}\right)^{\frac{1}{2}}$ then;

$$
\begin{gathered}
\frac{\partial \phi}{\partial x}=\frac{d \phi}{d \eta} \frac{\partial \eta}{\partial x}=\frac{1}{4} \sqrt{\frac{2}{\in}} \frac{\left(x-\left(x^{2}+y^{2}\right)^{\frac{1}{2}}-x\right)^{\frac{1}{2}}}{\left(\left(x^{2}+y^{2}\right)^{\frac{1}{2}}-x\right)^{\frac{1}{2}}\left(x^{2}+y^{2}\right)^{\frac{1}{2}}} \frac{d \phi}{d \eta} \\
\frac{\partial \phi}{\partial y}=\frac{d \phi}{d \eta} \frac{\partial \eta}{\partial y}=\frac{1}{4} \sqrt{\frac{2}{\epsilon}} \frac{y}{\left(\left(x^{2}+y^{2}\right)^{\frac{1}{2}}-x\right)^{\frac{1}{2}}\left(x^{2}+y^{2}\right)^{\frac{1}{2}}} \frac{d \phi}{d \eta} \\
\frac{\partial \phi}{\partial x^{2}}=\frac{d^{2} \phi}{d \eta^{2}}\left(\frac{\partial \eta}{\partial x}\right)^{2}=\frac{1}{8 \in} \frac{\left(x-\left(x^{2}+y^{2}\right)^{\frac{1}{2}}\right)^{2}}{\left(\left(x^{2}+y^{2}\right)^{\frac{1}{2}}-x\right)\left(x^{2}+y^{2}\right)} \frac{d^{2} \phi}{d \eta^{2}} \\
\frac{\partial \phi}{\partial y^{2}}=\frac{d^{2} \phi}{d \eta^{2}}\left(\frac{\partial \eta}{\partial y}\right)^{2}=\frac{1}{8 \in\left(\left(x^{2}+y^{2}\right)^{\frac{1}{2}}-x\right)\left(x^{2}+y^{2}\right)} \frac{y^{2}}{d \eta^{2}}
\end{gathered}
$$

Substituting $(11),(12),(13),(14)$ and $(15)$ into $\frac{d \phi}{d \eta}=c e^{-\eta^{2}}$
(9) we have:

$\frac{d^{2} \phi}{d \eta^{2}}+\left(\frac{\left(x^{2}+y^{2}\right)-x}{2 \in}\right)^{\frac{1}{2}} \frac{d \phi}{d \eta}=0$

i.e

$\frac{d^{2} \phi}{d \eta^{2}}+2 \eta \frac{d \phi}{d \eta}=0$

Let:

$\frac{d \phi}{d \eta}=p$

then;

$\frac{d p}{d \eta}+2 \eta p=0$

i.e

$p=c e^{-\eta^{2}}$ $\phi(\eta)=c \int_{0}^{\eta} e^{-z^{2}} d z+c_{1}$

$\phi(0)=1 \Rightarrow c_{1}=1$

$\phi(\infty)=0 \Rightarrow c \int_{0}^{\infty} e^{-z^{2}} d z+1=0$

i.e;

$$
\begin{aligned}
& c \frac{\sqrt{\pi}}{2}+1=0 \Rightarrow c=-\frac{2}{\sqrt{\pi}} \\
& \phi(\eta)=1-\frac{2}{\sqrt{\pi}} \int_{0}^{\eta} e^{-z^{2}} d z
\end{aligned}
$$

By definition:

$$
\frac{2}{\sqrt{\pi}} \int_{0}^{\eta} e^{-z^{2}} d z=\operatorname{erf}(\eta)
$$




$$
\begin{aligned}
& 1-\operatorname{erf}(\eta)=\operatorname{erfc}(\eta) \\
& \phi(\eta)=\operatorname{erfc}(\eta)
\end{aligned}
$$

abnd we obtain

\section{RESULT AND DISCUSSION}

Two dimensional advection dispersion equations describing the contaminant transport are solve analytically using the change of variable method. Analytical solution of equation (9) is computed.

The following figure explains contaminant concentration against the different parameters.

Figure 1 display the graph of contaminant concentration against distance $\mathrm{x}$ when peclet number is less than one. It shows that Contaminant concentration increases along $\mathrm{x}$ direction, but decreases as Peclet numbers increases. The real life interpretation here is that the dispersion is noticeably larger than advection, thus dispersion dominates and advection is negligible and this implies that contaminant concentration moves slowly to receptor.

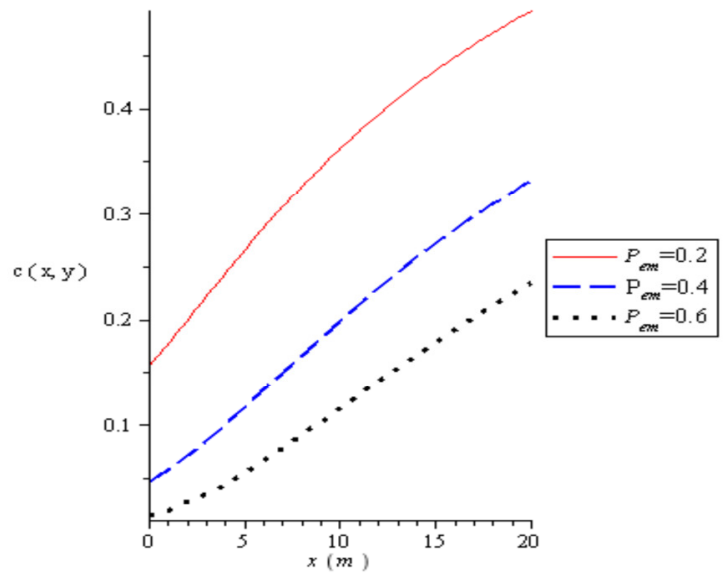

Fig 1: Graphical Illustration of Contaminant Concentration against distance travel for various values of Peclet number less than one

Figure 2 display the graph of contaminant concentration against distance $y$. It shows that Contaminant concentration decreases along $y$ direction, but decreases as Peclet numbers increases. The real life interpretation here is that the dispersion is noticeably larger than advection along y direction, thus dispersion dominates and advection is negligible along $\mathrm{y}$ direction and this implies that contaminant concentration moves slowly to receptor. $\phi(x, y)=\operatorname{erfc}\left(\frac{\left(x^{2}+y^{2}\right)^{\frac{1}{2}}-x}{2 \in}\right)^{\frac{1}{2}}$

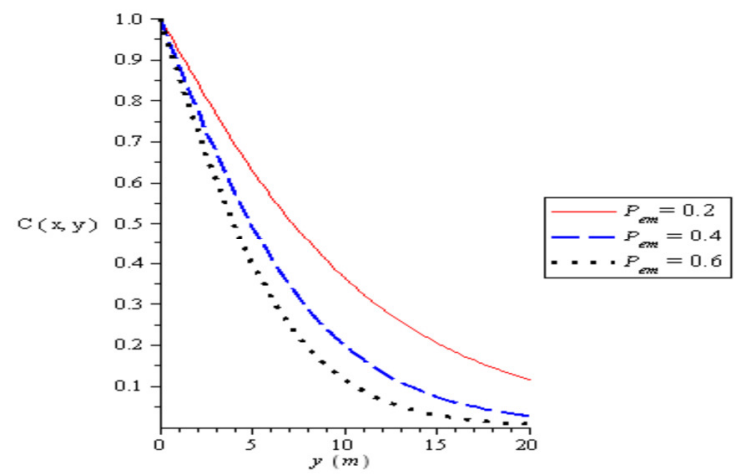

Fig.2: Graphical Illustration of Contaminant Concentration against distance travel for various values of Peclet number less than one.

Figure 3 display the graph of contaminant concentration against distance $\mathrm{x}$ and $\mathrm{y}$. It shows that Contaminant concentration increases along $\mathrm{x}$ direction and decreases along $y$ direction but decreases as peclet numbers increases. The real life interpretation here is that the dispersion is noticeably larger than advection along $\mathrm{x}$ and $\mathrm{y}$ direction, thus dispersion dominates and advection is negligible alongx and $\mathrm{y}$ direction and this implies that contaminant concentration moves slowly to receptor in both directions.

Figure 4 display the graph of contaminant concentration against distance $x$. It shows that Contaminant concentration increases along $\mathrm{x}$ direction, but decreases as Peclet numbers increases. The real life interpretation here is that the advection is noticeably larger than dispersion along $\mathrm{x}$ direction, thus advection dominates and dispersion is negligible and this implies that contaminant concentration moves faster along $\mathrm{x}$ direction to receptor. 


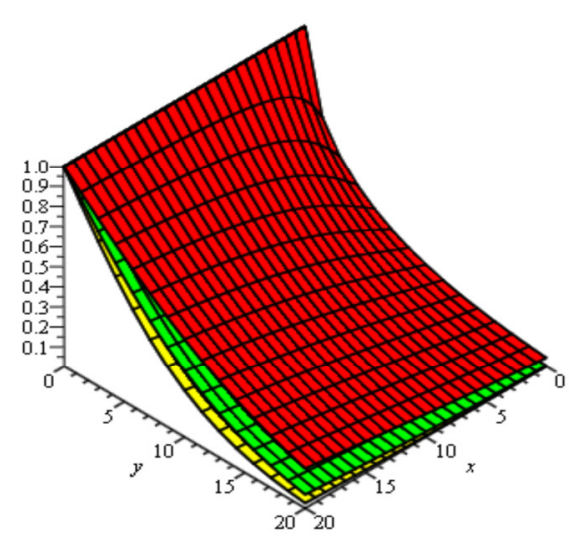

Fig3: Graphical Illustration of Contaminant Concentration against distances travel for various values of Peclet number less than one.

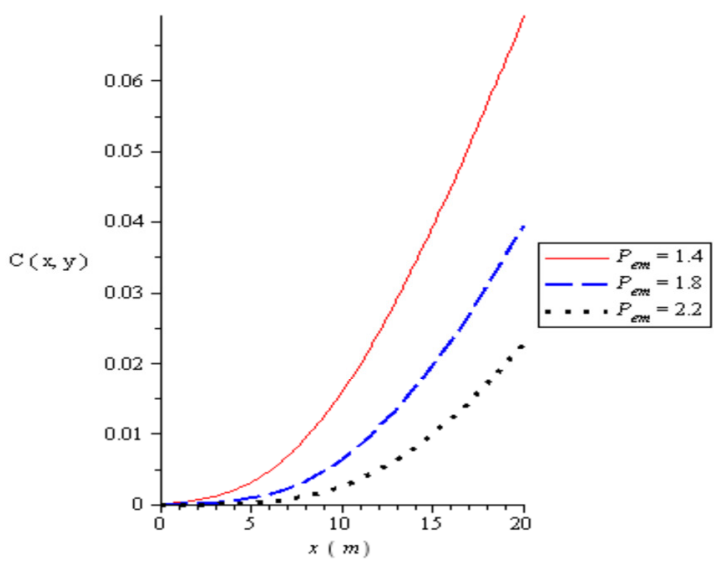

Fig 4: Graphical Illustration of Contaminant Concentration against distance travel for various values of Peclet number greater than one

Figure 5 display the graph of contaminant concentration against distance y. It shows that Contaminant concentration decreases along y direction, but decreases as Peclet numbers increases. The real life interpretation here is that the advection is noticeably larger than dispersion along y direction, thus advection dominates and dispersion is negligible and this implies that contaminant concentration moves faster along $y$ direction to receptor.

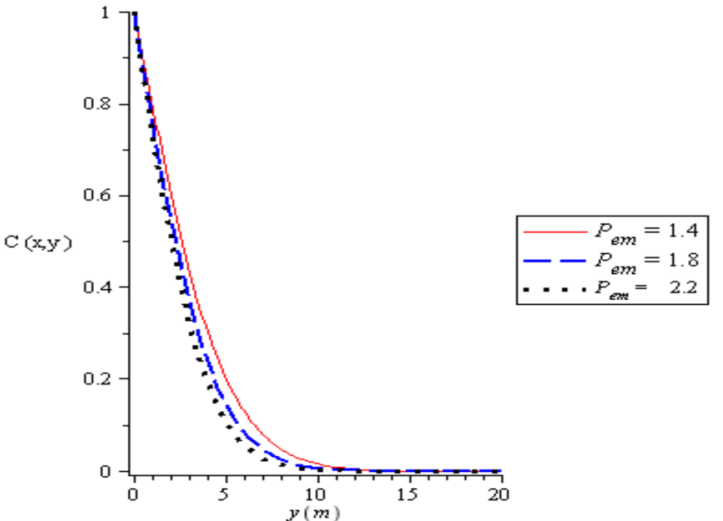

Fig 5: Graphical Illustration of Contaminant Concentration against distance travel for various values of Peclet number greater than one.

Figure 6 display the graph of contaminant concentration against distance $\mathrm{x}$ and $\mathrm{y}$. It shows that Contaminant concentration increases along $\mathrm{x}$ direction and decreases along $\mathrm{y}$ direction but decreases as peclet numbers increases. The real life interpretation here is that the advection is noticeably larger than dispersion along $\mathrm{x}$ and $\mathrm{y}$ direction, thus advection dominates and dispersion is negligible in both direction and this implies that contaminant concentration moves faster along $\mathrm{x}$ and $\mathrm{y}$ direction to receptor.

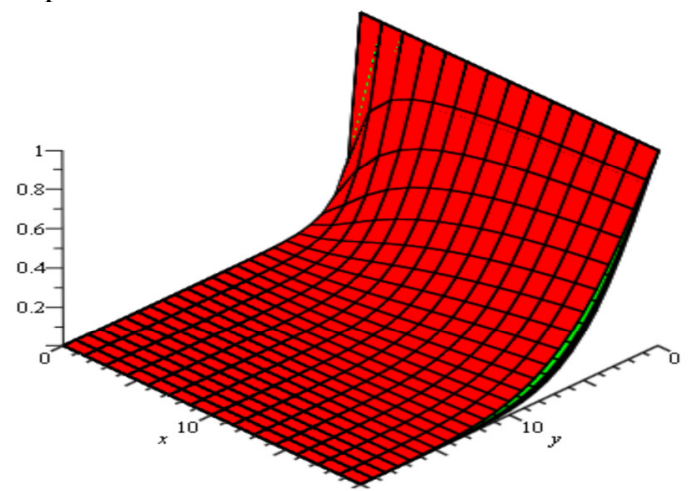

Fig 6: Graphical Illustration of Contaminant Concentration against distance travel for various values of Peclet number greater than one

Conclusions: We concluded that the contaminant concentration in two directions moves either fast or slow to receptor as affected by the values of Peclet number. The relative error committed in the solution by so doing is expected to be on the order of the Peclet number, and the smaller Peclet number, the smaller the error when peclet number is less than one, on the other hand, The relative error perpetrated in the solution by so doing is expected to be on the 
order of the inverse of the peclet number $\frac{1}{p e}$ and the larger $p e$, smaller the error for peclet number greater than one.

\section{REFERENCES}

Fetter, CW (1993). Contaminant Hydrology. New York: Macmillan Publishing co., p.2-38

Adeboy et al., (2014). Optimal Analysis of Contaminant Invasion in an Unconfined Aquifer System. International Journal of Science and Technology, 3(3), 184 -187.

Aiyesimi, Y. M; (2004). The Mathematical Analysis of Environmental Pollution of the Freudlich Non - linear Contaminant Transport Formulation. Journal of Nigerian Association of Mathematical Physics, 8(2), 8386
Patil, and Chore; (2014). ContaminantTransport through Porous Medial. An overview of Experimental and Numerical Studies. Journal of advances in Environmental Research

, 3(1), $45-69$

Fetter, C.W; (1993). Contaminant Hydrology. New York: Macmillan Publishing co., p. 2-38

Shehu, M.D; (2014). Mathematical Model for Optimal Control of Contaminants in an Aquifer. Unpublished P.hd Thesis from Federal University of Technology, Minna.

Mulligan, and Charette, (1998). Inter comparison Of Submarine Groundwater Discharge Estimate from a Sandy Unconfined Aquifer. Journal of Northeastern Geology and Environmental Science 26(2), p. 188-201 